\title{
Optimization of Extraction Method for GC-MS based Metabolomics for Filamentous Fungi
}

\author{
Siribhorn Madla1, Daisuke Miura² and Hiroyuki Wariishi' ${ }^{2,3 *}$ \\ ${ }^{1}$ Graduate School of Bioresource and Bioenvironmental Sciences, Kyushu University, 6-10-1 Hakozaki, Higashi-ku, Fukuoka, 812-8581, Japan \\ 2Innovation Center for Medical Redox Navigation, Kyushu University, 3-1-1 Maidashi, Higashi-ku, Fukuoka, 812-8582, Japan \\ ${ }^{3}$ Faculty of Agriculture, Kyushu University, 6-10-1 Hakozaki, Higashi-ku, Fukuoka, 812-8581, Japan
}

\begin{abstract}
Metabolite extraction is considered as one of the most important for quality controlling steps in metabolomic research. In the present study, optimal extraction protocol for fungal intracellular metabolites was investigated. Two extraction protocols with different solvent systems, methanol extraction and biphasic extraction under different $\mathrm{pH}$ conditions were compared. Number of detected peaks, sample-to-sample variation, and throughput of whole process were taken into account as criteria. Extracted metabolites were analyzed by gas chromatography-mass spectrometry (GC-MS) and principal component analysis (PCA). The extraction protocol using methanol as extraction solvent provided the highest number of detected peaks ( 300 peaks) with the ease and speed, suggesting that the methanol-extraction method was suitable for intracellular metabolites extraction from $P$. chrysosporium. However, depending on the nature of metabolites of interest, the biphasic extraction method under different $\mathrm{pH}$ conditions is suitable for a targeted analysis.
\end{abstract}

Keywords: Basidiomycetes; Extraction protocol; Optimization; Metabolomics

\section{Introduction}

Metabolome is complex because of the highly chemically and physically diverse nature of metabolites and of often occurring the multiplication of metabolic pathways for some particular metabolites. To obtain as much information about the metabolome as possible, using of productive sample preparation, merged with selective extractions associated with different analytical techniques and combined with the efficient mathematical interpretation of the data are crucial to examine $[1,2]$. Furthermore, the method should be optimized for each type of sample organisms of interest. Thus, the suitable selection for extraction protocols is considered as one of the most important quality controlling steps in microbial metabolome studies.

Mass spectrometry coupled with chromatography has become the preferred technique available for the quantification of metabolome. These analytical techniques possess high sensitivity, selectivity and simultaneous quantification of many different metabolites and require only a small sample volume for analysis [3,4]. In metabolomics, the number and amounts of metabolites extracted must be maximized, while the degradation of metabolites during sample preparation process and the analytical variation should be minimized [5]. Gas chromatography-mass spectrometry (GC-MS) is one of the most commonly used analytical techniques with a high liability and a capability of high-throughput and automated analysis.

Recently, the interest of using filamentous fungi such as Ascomycetes and Basidiomycetes as microbial cell factories to produce high-valued metabolites has been increased. Several researches about extraction strategy for intracellular metabolite of fungi were reported, especially in yeast [6-10], but only little is known about metabolites and their dynamics in filamentous fungi. Hence, the development of reliable method for metabolites determination in the organism has been prompted [11,12].

The white-rot Basidiomycete, Phanerochaete chrysosporium, has served as a model organism for studies of lignin biodegradation [13].
One possible target function is a fungal degradation of lignin which is well known to retard effective bio-energy production from woody materials. Effective and comprehensive extraction of intracellular metabolites from P. chrysosporium is difficult because this fungus has robust cell wall and very thick extracellular glucan layer [14].

In this study, different extraction methods were examined for its suitability for GC-MS based metabolomics in P. chrysosporium. Aiming to develop an extraction protocol for filamentous fungi with maximizing the number and amount of metabolites, whereas minimizing the analytical variation. GC-MS was utilized in the present work as an analytical apparatus because of its advantages for quick identification of low-molecular-weight metabolites based on retention times and fragmentation patterns. Optimized extraction protocol for $P$. chrysosporium would be applicable for other filamentous fungi.

\section{Materials and Methods}

\section{Chemicals}

All chemicals were of analytical grade and purchased from Wako Pure Chemicals Industries, Ltd, except $N$-methyl- $N$-trifluoroacetamide (MSTFA) from Thermo Fisher Scientific Inc. Deionized water was obtained with a Milli Q system (Millipore).

\section{Microorganism and culture conditions}

The culture medium (HCLN) containing $1 \%$ glucose, $1.2 \mathrm{mM}$

*Corresponding author: Hiroyuki Wariishi, 6-10-1 Hakozaki, Higashi-ku Fukuoka 812-8581, Kyushu University, Japan, Tel: 81-92-642-2992; E-mail: hirowari@agr.kyushu-u.ac.jp

Received December 19, 2011; Accepted January 19, 2012; Published January 24, 2012

Citation: Madla S, MiuraD, Wariishi H (2012) Optimization of Extraction Method for GC-MS based Metabolomics for Filamentous Fungi. J Microbial Biochem Technol 4: 005-009. doi:10.4172/1948-5948.1000064

Copyright: ( 2012 Madla S, et al. This is an open-access article distributed under the terms of the Creative Commons Attribution License, which permits unrestricted use, distribution, and reproduction in any medium, provided the original author and source are credited 
ammonium tartrate, $20 \mathrm{mM}$ dimethylsuccinate and 10\% (v/v) Kirk salts [15]. P. chrysosporium was grown on PDA slant for 7 days at $37^{\circ} \mathrm{C}$, then, $1 \%$ conidial suspension was inoculated to HCLN medium $(20 \mathrm{~mL}$ in a 200-mL Erlenmeyer flask) and grown at $37^{\circ} \mathrm{C}$ under static conditions. After a 2-day preincubation, mycelium mat was homogenized and transferred into $1 \mathrm{~L}$ production medium in a $2 \mathrm{~L}$ Erlenmeyer flask and incubated at $37^{\circ} \mathrm{C}, 140 \mathrm{rpm}$ for 4 days under air.

\section{Quenching of cellular metabolism}

One volume of culture broth was quickly harvested and filtered through a membrane filtration $(0.45 \mu \mathrm{m})$ and washed twice with deionized water. Wet mycelium was then mixed with 1 volume of $50 \%$ cold methanol $\left(-30^{\circ} \mathrm{C}\right)$. After quenching, wet mycelium was centrifuged ( $5 \mathrm{~min}, 11900 \times g$ ) at $-20^{\circ} \mathrm{C}$ and ground to powder by using a liquid $\mathrm{N}_{2}$ cooled pestle and mortar prior to freeze dry. Freeze-dried sample was stored at $-80^{\circ} \mathrm{C}$.

\section{Intracellular metabolites extraction}

Dried mycelium was mixed with small amount of sea sand (2035 mesh), ground to powder by using a liquid $\mathrm{N}_{2}$-cooled pestle and mortar. A brown-glass centrifuge tube containing $30 \mathrm{mg}$ of powdered mycelium was mixed with internal standards; $50 \mu \mathrm{L}$ of a ribitol stock solution $(0.2 \mathrm{mg} / \mathrm{mL})$ and $50 \mu \mathrm{L}$ of a nonadecanoic acid methyl ester stock solution $(2 \mathrm{mg} / \mathrm{mL})$. Four different extraction protocols were used and experiment was replicated five times in each extraction protocol. The relative concentration of intracellular metabolites were determined and compared.

\section{Methanol extraction}

Sample was extracted with $1.5 \mathrm{~mL}$ cold methanol $\left(-30^{\circ} \mathrm{C}\right)$, vigorously mixed, sonicated and centrifuged $(20 \mathrm{~min}, 100 \mathrm{x} g$ ) at room temperature. The supernatant was transferred to a new vial and the precipitated pellet was re-extracted with $1.5 \mathrm{~mL}$ of cold methanol. All extracts were pooled, frozen in liquid $\mathrm{N}_{2}$, concentrated by freeze-dryer, and stored at $-80^{\circ} \mathrm{C}$.

\section{Biphasic extraction under neutral conditions}

The final ratio of methanol-chloroform-water extraction method was 1:1:0.9 [16]. Sample was homogenized twice in $1.5 \mathrm{~mL}$ cold methanol $\left(-30^{\circ} \mathrm{C}\right), 0.75 \mathrm{~mL}$ cold chloroform $\left(-30^{\circ} \mathrm{C}\right)$ and $0.6 \mathrm{~mL}$ cold water $\left(4^{\circ} \mathrm{C}\right)$ to reach the first ratio of 1:0.5:0.4. Then, another $0.75 \mathrm{~mL}$ cold chloroform was added and mixed to form the second ratio of 1:1:0.4. Finally, $0.75 \mathrm{~mL}$ cold water was added to get the final ratio of 1:1:0.9, and samples were vortexed and centrifuged (10 $\mathrm{min}, 1000 \mathrm{x}$ $g$ ) at room temperature. The upper phase (methanol and water) was separated from the lower phase (chloroform) using a glass syringe, transferred to a new vial independently. Both fractions were frozen in liquid $\mathrm{N}_{2}$, freeze-dried, and stored at $-80^{\circ} \mathrm{C}$ until further analysis.

\section{Biphasic extraction under acidic conditions}

The extraction protocol was the same as previously described in methanol-chloroform-water extraction under neutral conditions. The acidic conditions were carried out with $0.1 \mathrm{M}$ formic acid. The upper phase containing methanol and formic acid aqueous solution ( $\mathrm{pH} \sim$ 3) was separated and then neutralized by the addition of $0.1 \mathrm{M} \mathrm{KOH}$ checking with $\mathrm{pH}$ test paper. The precipitated salts were removed by centrifugation and the supernatant was frozen in liquid $\mathrm{N}_{2}$, freezedried, and stored at $-80^{\circ} \mathrm{C}$ until further analysis.

\section{Biphasic extraction under basic conditions}

The extraction protocol was the same as previously described in methanol-chloroform-water extraction under neutral conditions. The alkaline extraction was carried out with $0.1 \mathrm{M} \mathrm{KOH}$. The upper phase compose of methanol and potassium hydroxide aqueous solution $(\mathrm{pH}$ $\sim 12$ ) was neutralized by the addition of $0.1 \mathrm{M}$ formic acid checking with $\mathrm{pH}$ paper and removed precipitated salts by centrifugation. The supernatant was frozen in liquid $\mathrm{N}_{2}$, freeze-dried, and stored at $-80^{\circ} \mathrm{C}$ until further analysis.

\section{GC-MS analysis}

O-Methylhydroxylamine Hydrochloride $(100 \mu \mathrm{L}$ of $30 \mathrm{mg} / \mathrm{mL}$ in dry-pyridine) was added to dried metabolites, incubated at $30^{\circ} \mathrm{C}, 200$ rpm for 120 minutes to increase the degree of methoximation, and then dried under $\mathrm{N}_{2}$ [17]. Then $100 \mu \mathrm{L}$ MSTFA and $50 \mu$ pyridine were added, incubated at $37^{\circ} \mathrm{C}$ for 90 minutes [18]. The derivatized samples were analyzed by GC-MS coupled with quadrapole detector (GCMSQP2010 plus, Shimadzu, Japan). A fused silica column of Rtx ${ }^{\mathbb{B}}-5 \mathrm{MS}$ (30 $\mathrm{m}$ x $0.25 \mathrm{~mm}$ i.d., $0.25 \mu \mathrm{m}$ film thickness, RESTEK) was used and temperature was programmed from 80 to $320^{\circ} \mathrm{C}$ at $8^{\circ} \mathrm{C} / \mathrm{min}$, and hold for 5 minutes. The injection temperature was $280^{\circ} \mathrm{C}$. Mass spectra were obtained with electron impact ionization at $70 \mathrm{eV}$ with the split ratio of $25: 1$.

\section{Data processing and statistical analysis}

The raw data files obtained from GC-MS runs were converted to vendor-independent NetCDF (network Common Data Format) format. MZmine software version 0.60 (http://mzmine.sourceforge. net/) was applied for data processing. MZmine processing consists of several stages, which are input file manipulation, spectral filtering, peak detection, chromatographic alignment, normalization, visualization and data export, each of which is controlled by several parameters. The preprocessed GC-MS data was then imported into SIMCA-P 11.5 software (Umetrics Inc., Umeå, Sweden). Principal component analysis (PCA) was applied to identify the degree of similarity between the data.

\section{Results and Discussion}

\section{Effect of biomass to methanol ratio}

Our previous data indicated that fungal biomass in the culture system causes minimal deviations during the early stage [19]. Effect of organic solvents on the amount of intracellular metabolites extracted from mycelium of $P$. chrysosporium was investigated. Among solvents tested, methanol was found to be the best based on the highest amount of extracts. Methanol has been reported to be a good organic solvent for extraction with many advantages; easy to remove after extraction, good recovery of metabolites, good reproducibility, and broad range of metabolites extractable [2]. To determine the best protocol, four biomass-to-methanol ratios; $2.5,5,10$ and $20 \mathrm{mg} / \mathrm{mL}$ were examined. As the ratios of biomass to methanol increased, the number of detected peaks was increased until it reached the maximum at the ratio of 10 $\mathrm{mg} / \mathrm{mL}$ (Table 1). Since one important goal in this study was to develop the extraction method which enables to extract the metabolites as many as possible. Therefore, the ratio of biomass to methanol at $10 \mathrm{mg} /$ $\mathrm{mL}$ was selected for further studies. The balance between extraction efficiency and occurrence of metabolite fragmentation should be considered for optimization of extraction protocols; however, we put 
priority to a number of metabolite-derived peaks at this stage, since a later analysis can point out the true metabolic data and fragment data but any analytical methods cannot discuss on a metabolite with no peak appearance.

\section{Evaluation of extractable metabolites}

The optimized extraction protocol should be developed for each microorganism of interest. Based on the concept that polar solvents extract polar metabolites and non-polar solvents extract non-polar metabolites; likewise, acid stable metabolites should be extracted with acids whereas alkali stable metabolites should be extracted with alkali [3]. Thus, the effect of solvents and $\mathrm{pH}$ conditions on metabolite extraction was investigated using $P$. chrysosporium.

Based on the number of detected peaks, polar phases contained more peaks than non-polar phases (Table 2). Biphasic extraction solvent system composed of methanol with $\mathrm{pH}$-controlled water to extract polar metabolites and chloroform for non-polar metabolites showing the possibility to separate polar from non-polar metabolites. These two phases can be extracted simultaneously and each fraction can be analyzed separately, which may cause better separation because of peak numbers and of column selection. For this reason, methanol/chloroform/water was previously reported as the preferred extraction method for lipid-rich tissues such as liver and brain [2022]. Phospholipids, a class of lipids and a major component of all cell membranes, can also cause ion suppression in electrospray ionization apparatus, which further prevents ionization and detection of other metabolites [21]. Hence, minimizing lipids contamination is necessary to prevent ion suppression and subsequent poor detection of other metabolites [22]. Moreover, chloroform presented in biphasic extraction solvent mixtures is able to denature of enzymes avoiding further reactions [2]. Therefore, considering on the possibility to remove polar out of non-polar metabolites, which is mainly referred to lipid compounds, the biphasic extraction under acidic conditions was preferable.

\section{Evaluation of different extraction protocols using PCA}

Polar phase: Principal components analysis (PCA) was used to

\begin{tabular}{|c|c|}
\hline Biomass/Methanol $(\mathrm{mg} / \mathrm{mL})$ & No. detected peaks $^{\mathrm{a}}$ \\
\hline 2.5 & $237 \pm 19$ \\
\hline 5 & $247 \pm 15$ \\
\hline 10 & $299 \pm 6$ \\
\hline 20 & $295 \pm 5$ \\
\hline
\end{tabular}

a Means \pm SD determined the total peak number with the peak height cut off at 12000.

Table 1: Comparison of total detected peak of intracellular metabolites extracted from different biomass to methanol ratios.

\begin{tabular}{|l|c|c|}
\hline Extraction method & $\begin{array}{c}\text { Number of detected peaks } \\
\text { in polar phase }\end{array}$ & $\begin{array}{c}\text { Number of detected peaks } \\
\text { a in } \\
\text { non-polar phase }\end{array}$ \\
\hline Methanol & 299 & No phase separation \\
\hline Biphasic-neutral & 210 & 151 \\
\hline Biphasic-acidic & 282 & 190 \\
\hline Biphasic-basic & 216 & 251 \\
\hline
\end{tabular}

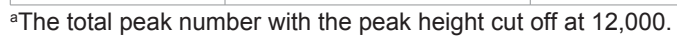

Table 2: Comparison of total detected peak numbers and the yields of intracellular metabolites extracted from different solvent systems.
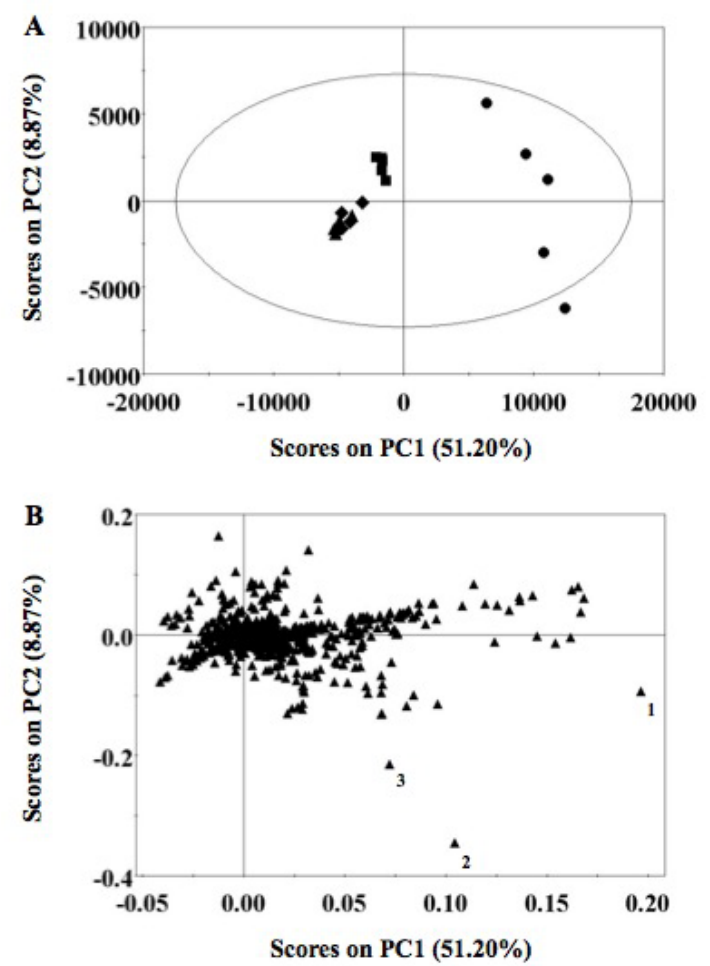

Figure 1: PCA scores plot $(A)$ and PCA loadings plot $(B)$ from the GC-MS spectra originated from polar phase of $P$. chrysosporium mycelium extracted by using different solvent systems $(\bullet)$ methanol, ( $\mathbf{\square})$ biphasic under neutral condition, $(\boldsymbol{\Delta})$ biphasic under acidic condition, and $(\boldsymbol{)})$ biphasic under basic condition. Metabolite assignments: (1) tetracosanoic acid; (2) hexadecanoic acid; (3) pentadecanoic acid

compare the sample-to-sample variation of metabolic fingerprints derived from 5 replicates in each different extraction protocols. Representative GC-MS spectra showing the metabolic fingerprints of mycelium extracts in polar phases including methanol presented in (Figure 1). From PCA scores plot (Figure 1A), methanol extraction method was clearly separated from biphasic extraction method explained by PC1. The sample-to-sample variation can be implied the reproducibility of the method, which is explained by PC2 of (Figure $1 \mathrm{~A})$, indicating that all four extraction methods demonstrated a good reproducibility since the contribution of PC2 was only $8.87 \%$ when the data separation was maximal. However, methanol extraction apparently showed the largest sample-to-sample variation among four tested extraction methods, as evidenced by lacking of a tight cluster formation in the PCA scores plot. As shown by the PCA loadings plot (Figure 1B), tetracosanoic acid, hexadecanoic acid and pentadecanoic acid are metabolites that contribute to the separation of methanol extraction data from biphasic clusters.

Furthermore, each peak in the chromatogram was putatively identified by using the data libraries and Venn diagram plot was applied to distinguish the unique metabolites presented in each extraction method. The result revealed that the sample via methanol extraction protocol contained a series of free fatty acids (ca. 30\% of total unique metabolites) which were not found in biphasic extraction methods (Figure 2).

Non-polar phase: Three sets of data from non-polar phase derived from biphasic extraction methods were further investigated 
Citation: Madla S, MiuraD, Wariishi H (2012) Optimization of Extraction Method for GC-MS based Metabolomics for Filamentous Fungi. J Microbial Biochem Technol 4: 005-009. doi:10.4172/1948-5948.1000064

for sample-to-sample variation of each condition by PCA. According to PCA scores plot (Figure 3A), three different $\mathrm{pH}$ conditions were distinct from each other, although some data points from neutral and acidic conditions were overlapped. Not like Fig. 2, PC2 was not the axis explaining the sample-to-sample variation. On the other hand,

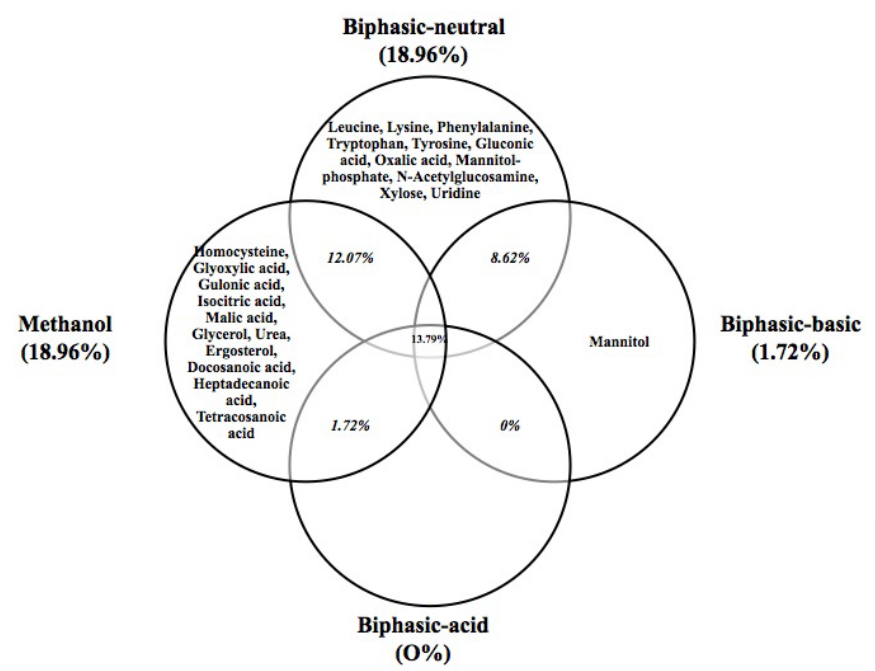

Figure 2: Venn diagram illustrates the overlap of intracellular metabolites extracted from mycelium of $P$. chrysosporium in polar phase with four different solvents extraction systems.
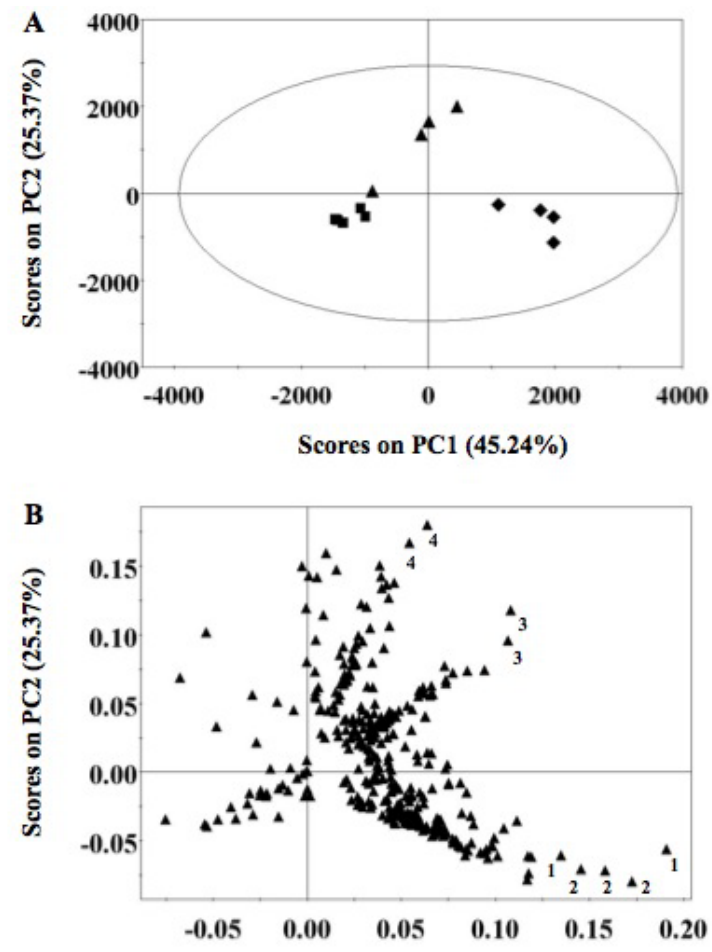

Scores on PC1 (45.24\%)

Figure 3: PCA scores plot (A) and PCA loadings plot (B) from the GC-MS spectra derived from non-polar phase of $P$. chrysosporium mycelium extracted by using different solvent systems ( $\mathbf{\square})$ biphasic under neutral condition, $(\boldsymbol{\Delta})$ biphasic under acidic condition, and ( $\bullet$ biphasic under basic condition Metabolite assignments: (1) oleic acid; (2) propanoic acid; (3) ergosterol; (4) 9,12-octadecadienoic acid (Z,Z).

\begin{tabular}{|l|c|c|c|c|c|}
\hline \multirow{2}{*}{$\begin{array}{c}\text { Extraction } \\
\text { method }\end{array}$} & \multicolumn{2}{|c|}{$\begin{array}{c}\text { Number of } \\
\text { detected peaks }\end{array}$} & \multicolumn{2}{|c|}{$\begin{array}{c}\text { Sample-to-sample } \\
\text { variation }\end{array}$} & \multirow{2}{*}{ Speed of the method } \\
\cline { 2 - 6 } & polar & Non-polar & polar & Non-polar & \\
\hline Methanol & \multicolumn{2}{|c|}{+++} & \multicolumn{2}{|c|}{ Small } & Fast and easy \\
\hline Biphasic-neutral & + & + & Small & Moderate & Time consuming \\
\hline Biphasic-acidic & +++ & ++ & Small & Moderate & Time consuming \\
\hline Biphasic-basic & ++ & +++ & Small & Moderate & Time consuming \\
\hline
\end{tabular}

anly one phase derived from methanol extraction solvent system $+++\quad$ the highest detected peak numbers (in this experiment) $++\quad$ the second high detected peak numbers (in this experiment) $++\quad$ the third high detected peak numbers (in this experiment) $+\quad$ the fourth high detected peak numbers (in this experiment)

Table 3: Summary of solvent extraction systems for intracellular metabolites extraction.

the PCA loading plot (Figure 3B) indicated that oleic acid, propanoic acid, ergosterol and 9, 12-octadecadienoic acid $(\mathrm{Z}, \mathrm{Z})$ were compounds conducing to the difference among three different extraction $\mathrm{pHs}$.

As shown in Table 3, methanol solvent system presented the highest number of detected peaks with a small sample-to-sample variation, representing the high reproducibility of the method. Furthermore, the advantages of this method were simple and fast. Biphasic extraction method under acidic and basic conditions also gave the high number of detected peaks in both polar and non-polar phases. The biphasic extraction showed the possibility to separate polar from non-polar metabolites, but the weak point of this method was the time consuming.

However, for metabolic fingerprinting studies, an acid and an alkaline extraction may contain advantages depending on the metabolite(s) of interest, but a number of compounds are unstable at high and low pHs (dihydroxyacetone phosphate (DHAP) and phospho(enol)pyruvate (PEP) are well-know examples) and are degraded $\left(\mathrm{NAD}^{+}\right.$in acid solution and $\mathrm{NADH}$ in alkaline solution) as previously described on the study of glycolytic intermediates levels in Aspergillus niger mycelium [9,12,23]. It was found that the extraction method using chloroform at neutral conditions appeared to be superior to both extractions with acidic and basic conditions, since larger amounts of glycolytic intermediates were extracted. They assumed that it was probably due to instability of these metabolites at high and low pHs [11]. Moreover, acid extraction, which is widely used in protein precipitation and hydrophilic metabolites extraction, is not only caused the damage of metabolite structures, but also make problematics for many analytical methods [20].

It would be noteworthy to mention that the fragmentation of several metabolites could be pointed out from the PCA loading plot seen as several points derived from one metabolite (Figures 1 and 2). However, this type of multiplication was quite similar among 4 protocols in the present study (data not shown), suggesting that the extent of fragmentation during extraction processes were similar. Furthermore, the thermal fragmentation may also occur at the injection port of GC.

\section{Conclusion}

Based on number of detected peaks, sample-to-sample variation, the ease and speed of the experimental efficiency, methanol extraction method is preferable and selected as the method for the extraction of 
Citation: Madla S, MiuraD, Wariishi H (2012) Optimization of Extraction Method for GC-MS based Metabolomics for Filamentous Fungi. J Microbial Biochem Technol 4: 005-009. doi:10.4172/1948-5948.1000064

intracellular metabolites from $P$. chrysosporium. However, depending on the nature of metabolites of interest, the biphasic extraction method under different $\mathrm{pH}$ conditions is an optional. Furthermore, pre-separation based on characteristics of metabolites may provide a better strategy for analytical optimization such as column selection for chromatography and ionization selection for MS analysis.

\section{Acknowledgement}

This research was supported by the Science and Technology Incubation Program in Advanced Region from the funding program "Creation of Innovation Centers for Advanced Interdisciplinary Research Areas" from Japan Science and Technology Agency, commissioned by the Ministry of Education, Cultures, Sports, Science and Technology. S.M. thanks the Japanese Government's Ministry of Education, Culture, Sports, Science and Technology (MEXT) for a PhD scholarship.

\section{References}

1. Hoque Md A, Ushiyama H, Tomita M, Shimizu K (2005) Dynamic responses of the intracellular metabolite concentrations of the wild type and pykA mutant Escherichia coli against pulse addition of glucose or $\mathrm{NH}_{3}$ under those limiting continuous cultures. Biochem Eng J 26: 38-49.

2. Villas-Bôas SG, Roessner U, Hansen MAE, Smedsgaard J, Nielsen J (2006) Sampling and sample preparation: Metabolome Analysis: An Introduction New Jersey, Hoboken.

3. Mashego MR, Rumbold K, De Mey M, Vandamme E, Soetaert W, et al. (2007) Microbial metabolomics: past present and future methodologies. Biotechnol Lett 29: 1-16.

4. Li X, Xu Z, Lu X, Yang X, Yin P, et al. (2009) Comprehensive two-dimensional gas chromatography/time-of-flight mass spectrometry for metabonomics: Biomarker discovery for diabetes mellitus. Anal Chim Acta 633: 257-262.

5. Gullberg J, Jonsson P, Nordström A, Sjöström M, Moritz T (2004) Design of experiments: an efficient strategy to identify factors influencing extraction and derivatization of Arabidopsis thaliana samples in metabolomic studies with gas chromatography/mass spectrometry. Anal Biochem 331: 283-295.

6. Gonzalez B, François J, Renaud M (1997) A rapid and reliable method for metabolite extraction in yeast using boiling buffered ethanol. Yeast 13: 13471355.

7. Lange HC, Eman M, van Zuijlen G, Visser D, van Dam JC, et al. (2001) Improved rapid sampling for in vivo kinetics of intracellular metabolites in Saccharomyces cerevisiae. Biotechnol Bioeng 75: 406-415.

8. Castrillo JI, Hayes A, Moammed S, Gaskell SJ, Oliver SG (2003) An optimized protocol for metabolome analysis in yeast using direct infusion electrospray mass spectrometry. Phytochem 62: 929-937.

9. Villas-Bôas SG, Højer-Pedersen J, Åkesson M, Smedsgaard J, Nielsen J (2005) Global metabolite analysis of yeast: evaluation of sample preparation methods. Yeast 22: 1155-1169.

10. Crutchfield CA, Lu W, Melamud E, Rabinowitz JD (2010) Mass spectrometrybased metabolomics of yeast. Methods Enzymol 470: 393-426.

11. Ruijter GJG, Visser J (1996) Determination of intermediary metabolites in Aspergillus niger. J Microbiol Methods 25: 295-302.

12. Hajjaj H, Blanc PJ, Goma G, Francois J (1998) Sampling techniques and comparative extraction procedures for quantitative determination of intra-and extracellular metabolites in filamentous fungi. FEMS Microbiol Lett 164: 195200.

13. Eggert C, Temp U, Eriksson KEL (1997) Laccase is essential for lignin degradation by the white-rot fungus Pycnoporus cinnabarinus. FEBS Lett 407 : 89-92.

14. Buchala AJ, Leisola MSA (1987) Structure of the ß-D-glucan secreted by Phanerochaete chrysosporium. Carbohydrate Res 165: 146-149.

15. Kirk TK, Schultz E, Connors WJ, Lorenz LF, Zeikus JG (1978) Influence of Culture Parameters on Lignin Metabolism by Phanerochaete chrysosporium. Arch Microbiol 117: 277-285.
16. Bligh EG, Dyer WJ (1959) A rapid method of total lipid extraction and purification. Can J Biochem Physiol 37: 911-917.

17. Kusano M, Fukushima A, Kobayashi M, Hayashi N, Jonsson P, et al. (2007) Application of a metabolomic method combining one-dimensional and twodimensional gas chromatography-time-of-flight/mass spectrometry to metabolic phenotyping of natural variants in rice. J Chromatogr B 855: 71-79.

18. Miura D, Tanaka H, Wariishi $\mathrm{H}$ (2004) Metabolomic differential display analysis of the white-rot basidiomycete Phanerochaete chrysosporium grown under air and $100 \%$ oxygen. FEMS Microbiol Lett 234: 111-116.

19. Shimizu M, Yuda N, Nakamura T, Tanaka H, Wariishi H (2005) Metabolic regulation at the tricarboxylic acid and glyoxylate cycles of the lignin-degrading basidiomycete Phanerochaete chrysosporium against exogenous addition of vanillin. Proteomics 5: 3919-3931.

20. Lin CY, Wu H, Tjeerdema RS, Viant MR (2007) Evaluation of metabolite extraction strategies from tissue samples using NMR metabolomics. Metabolomics 3: 55-67.

21. Wu H, Southam AD, Hines A, Viant MR (2008) High-throughput tissue extraction protocol for NMR- and MS-based metabolomics. Anal Biochem 372: 204-212.

22. Le Belle JE, Harris NG, Williams SR, Bhakoo KK (2002) A comparison of cell and tissue extraction techniques using high-resolution ${ }^{1} \mathrm{H}-\mathrm{NMR}$ spectroscopy. NMR Biomed 15: 37-44.

23. de Koning W, van Dam K (1992) A method for the determination of changes of glycolytic metabolites in yeast on a subsecond time scale using extraction at neutral pH. Anal Biochem 204: 118-123. 